\title{
Analysis on Influencing Factors of Residents' Satisfaction with Waste Classification Effect in Beijing
}

\author{
Han Song, Li Haoyu* \\ Data Science and Analytics, Beijing Wuzi University, Beijing, China \\ Email address: \\ 18501982239@163.com (Li Haoyu) \\ ${ }^{*}$ Corresponding author
}

To cite this article:

Han Song, Li Haoyu. Analysis on Influencing Factors of Residents' Satisfaction with Waste Classification Effect in Beijing. International Journal of Data Science and Analysis. Vol. 7, No. 5, 2021, pp. 122-131. doi: 10.11648/j.ijdsa.20210705.12

Received: September 23, 2021; Accepted: October 14, 2021; Published: October 21, 2021

\begin{abstract}
This survey aims to explore the evaluation of influencing factors of Beijing residents' satisfaction with the effect of waste classification since the implementation of the regulations. The questionnaire was distributed to all residents in Beijing by means of probability and non-probability sampling. Sixteen districts and counties in Beijing were divided into five major districts according to their functions as first-level sampling units. Finally, a total of 1307 questionnaires were collected to explore the influencing factors of residents' satisfaction with waste classification with the help of random forest model. On this basis, ten influencing factors affecting residents' satisfaction with waste classification were found. At the same time, combined with the suggestions given by residents on waste classification in the questionnaire, the effective information is intuitively observed through the text analysis results to see what factors improve residents' satisfaction with waste classification. Through the above research, we find that the residents' cognitive level of waste classification and the evaluation of the implementation of waste classification policies are the main reasons for the low residents' satisfaction with waste classification. In addition, communities have timely formulas for waste classification policies, clear rewards and punishments, and solve the problems of weak publicity and lax supervision to improve residents' satisfaction in waste classification.
\end{abstract}

Keywords: Waste Classification, Classification Effect Satisfaction, Random Forests, The Text Analysis

\section{Introduction and Review}

In order to improve the efficiency of waste disposal and reduce the amount of public resources consumed by landfills every year, Beijing has officially implemented the Beijing Municipal Household Waste Management Regulations. Since its implementation one year ago, the regulations have not only brought earth-shaking changes to Beijing's environment, but also provided a platform for the popularization of waste classification knowledge in the city. With the rapid development of economy and society, the continuous improvement of people's living standard and the acceleration of urbanization, the production of municipal solid waste is also rising sharply. In 2019, domestic waste production in 337 first-tier to fifth-tier cities reached 343 million tons. Among them, Beijing municipal household waste production is about 9.6 million tons, accounting for $2.80 \%$ of the total national household waste production, is expected to exceed
10 million tons in 2020.

The treatment, disposal and resource utilization of municipal solid waste are related to national economy and people's livelihood and environmental protection. The goal or principle of waste disposal is harmlessness, reduction and resource. Household waste classification is one of the preconditions to realize the harmless treatment of urban household waste. To improve the efficiency of waste recycling, it is necessary to classify waste in advance. For example, classifying kitchen waste and dead leaves as compost can improve the quality of compost. Waste batteries, waste lamp tubes and other harmful waste classification, can reduce the release of toxic substances to the environment pollution. Waste classification can improve the recycling rate of waste, so as to greatly reduce the amount of waste for terminal treatment and achieve the purpose of waste reduction. Waste classification is the focus of waste disposal in the future, and also an important measure to be implemented in urban waste control in China. 
In May 2020, the Beijing Municipal Household Waste Management Regulations officially came into effect, marking that the whole-process household waste classification management has entered a new stage of acceleration in accordance with the law. With the expanded year by year, Beijing urban construction waste disposal has become the chief problem of environment pollution in Beijing, the increase of waste and waste classification is not scientific, cause for burning and landfilling takes up a lot of land, at the same time there is a risk of secondary pollution, waste disposal technology rely on burning and burying is a drop in the bucket. Low efficiency of waste disposal can be effectively solved by controlling the source of waste generation and standardizing the treatment of different types of waste. Since the implementation of the regulations one year ago, how to implement the regulations more effectively and improve residents' satisfaction with the waste classification policy will be a thorny problem urgently to be solved by government managers.

In recent years, a large number of pilot demonstration and practical exploration of waste classification have been carried out across the country, and innovative attempts have been made in urban cleanliness and sanitation. At the same time, research on waste sorting is pouring out. In the existing literature on factors affecting residents' satisfaction with waste classification, most of the studies mainly focus on the influence of a single factor or the influence of some factors.

In terms of influencing the intention of waste classification, Li Wei et al. used hierarchical regression analysis and bootstrap method to find that propaganda and education would positively affect the emotion of waste classification (shame and pride of not practicing waste classification) [1]. Based on the self-construction theory, Hu Changchun et al. found that the type of waste classification propaganda and the group size of the audience when they contact the propaganda can adjust the satisfaction of waste classification [2]. Chen Jian et al. used TPB theory and Probit regression model to find that residents' satisfaction with waste classification has a significant impact on the awareness rate of waste classification, government publicity, government job satisfaction and facilities, and residents' classification behavior is significantly affected by waste classification intention and facilities [3].

In terms of the effect evaluation of waste classification, $\mathrm{Xu}$ Qianqian et al. measured the efficiency of rural household waste classification and treatment with the participation of farmers based on DEA, and used Tobit model for empirical analysis to analyze the factors affecting the efficiency of rural household waste classification and treatment. It is found that the awareness rate, participation status and supervision behavior of villagers in waste classification have a significant positive impact on the efficiency of waste classification and treatment [4]. Wang Qiqi conducted dummy variable regression analysis and descriptive statistical analysis to evaluate the implementation effect of waste classification in Shanghai [5]. Zhao Junqing believes that it is often difficult for residents to make quantitative judgment on the evaluation of the effect of waste classification, which is the main uncertainty factor affecting the evaluation of waste classification effect [6]. Musee et al. used the fuzzy classification algorithm for garbage, which combined the exposure and quantity factors of garbage, so as to reflect the ecological toxicity of garbage, so as to improve the garbage classification effect [7].

Through the discussion above similar studies on waste classification, we found that most of the existing studies on the satisfaction of waste classification effect mainly focus on the willingness of waste classification and the evaluation of waste classification effect, and most of the studies involve the impact cycle [8] and toxicity [9] of a kind of material waste. If the two aspects are studied separately, the research results will be more one-sided, and the conclusions are different from the actual residents' satisfaction with the effect of waste classification. Moreover, there are shortcomings in the analysis and processing of sample data, which are mostly limited to three statistical analysis methods of principal component analysis [10], regression analysis and descriptive statistics [11], so that the research results of waste classification effect satisfaction are often different from the actual results.

Based on this, this paper conducted a comprehensive survey of residents' satisfaction with waste classification in Beijing by combining the two factors that affect the satisfaction with waste classification effect. Random forest method was used to analyze the factors affecting the satisfaction of waste classification. Finally, combined with data mining method, the real feelings and thoughts of Beijing residents on waste classification are analyzed. During the survey, we divided the two influential factors of waste classification intention and waste classification effect into four indicators, namely, classification effect, management work, living environment and environmental management, to explore the influence of these four indicators on the difference of residents' satisfaction with waste classification in different functional areas [12].

\section{Research Design and Research Methods}

\subsection{Research Object}

The survey targets all residents of Beijing. Use access survey method and brainstorming method, randomly selected from 16 counties of residents, residents' committees and the relevant person in charge of the sanitation department, by the combination of online and offline access, further understand residents problems encountered during the outbreak of waste classification of and feelings, to get a general idea of the Beijing residents satisfaction they sort the waste, Lay the foundation for the design of the questionnaire. Finally, 1400 questionnaires were distributed online, 1307 of which were valid. The effective rate of sample was $93.4 \%$. 


\subsection{Sample Plan Design}

According to relevant information, the total resident population of Beijing was 21.536 million by the end of 2019 [13]. The formula was adopted by taking 16 municipal districts of Beijing as sampling boxes to calculate the sample size.

$$
n=\frac{N Z_{\frac{\alpha}{2}}^{2} S^{2}}{N d^{2}+N Z_{\frac{\alpha}{2}}^{2} S^{2}}
$$

According to the pre-survey data, the maximum variance calculated with SPSS is $S^{2}=1.259$, so that the relative error limit does not exceed $7.5 \%$ (that is, the absolute error limit $\mathrm{d}=0.15$ ), and the confidence level is $99 \%$. The initial sample size calculated is 366.24 , and the effective response rate of the pre-survey questionnaire is $91.7 \%$. Further adjustment of the sample size:

$$
n_{1}=\frac{n}{r_{1}} \approx 400
$$

Considering the sampling effect of improbability sampling, the calculation results were expanded 3.5 times and the sample size was finally determined to be 1400 samples. The questionnaire was distributed online during the survey, so the questionnaire recovery rate was $100 \%$. However, some respondents gave contradictory answers or did not answer the questions according to the questionnaire, so 93 questionnaires were invalid, with an effective questionnaire rate of $93.4 \%$.

\subsection{According to the Test Data}

In the examination of the formal survey data, we also conducted the discrimination test for each item of each scale first. After adding the scores of all items in the scale filled by each respondent to obtain the total score, they were sorted in descending order, with the top $27 \%$ as the high group and the bottom $27 \%$ as the low group. Then, independent sample $t$ test was conducted for each item scores of the high and low groups to judge the significance. According to the analysis results, the score of high sub group is above 107, and the score of low sub group is below 92. All the 16 items in the scale have the degree of differentiation, which can identify different interviewees.

Then, the reliability test is carried out on the sample data, and the results of each item are calculated as shown in the table. The analysis results show that the Cronbach $\alpha$ coefficient of each item is above 0.7 , so the reliability of the scale is good.

Table 1. Reliability test table of formal investigation data.

\begin{tabular}{llll}
\hline level & Cronbach $\alpha$ & A number of & The results of \\
\hline Willingness of waste classification & 0.719 & 3 & General \\
Waste sorting process & 0.854 & 6 & Very good \\
Effectiveness of waste separation & 0.777 & 3 & Good \\
Effect evaluation of waste classification & 0.827 & 4 & Very good \\
\hline
\end{tabular}

In terms of content validity, the scores of all factors and total table are significant, indicating that questionnaire Q14 has good content validity. The KMO coefficient of the scale is 0.904 , and the $\mathrm{P}$ value is 0.000 , indicating that the questionnaire has a good structural design, which means that the questionnaire has structural validity.

Table 2. Formal survey data KMO and Bartlett sphericity test results.

\begin{tabular}{lll}
\hline Adequacy test & KMO value & $\mathbf{0 . 9 0 4}$ \\
\hline \multirow{2}{*}{ Bartlett sphericity } & The approximate chi-square & 7161.478 \\
test & Degrees of freedom & 120 \\
& P value & 0.000 \\
\hline
\end{tabular}

\subsection{Research Tools}

This paper mainly studies the factors influencing the difference of satisfaction degree of waste classification effect among Beijing residents since the implementation of waste classification policy one year ago. Among them, waste classification intention, waste classification process, waste classification effect and waste classification effect evaluation are designed from multiple aspects. Moreover, multicollinearity exists among questions within each variable in the questionnaire, which will affect the accuracy of the final research results. Therefore, the random forest model was selected in this paper to solve the above problems, and the word frequency analysis was used to verify which factors would affect the satisfaction of waste classification effect of Beijing residents from the side.

Random forest is a machine learning method proposed by Breiman et al., which is a decision tree-based classifier with Boosting and SVM classification effect. Under the condition of without any increase in the original sample set samples by pulling boots for build a set of sample subset selection component classifier, and then using the results of the voting system integrated component classifier to get the final classification results, when building component classifier, not outside of selected sample bag (OOB) data sets, with a bag of error test data get the bag. In addition to have no need for data preprocessing, the more problems dealing with the advantages of convenient and quick, stable classification results, the characteristics of random forest is an important is can be realized in the process of sample training characteristic of the importance of evaluation, the importance of the characteristics of $\mathrm{f}$ through after immediately to exchange the sampling characteristics in OOB, statistical classification accuracy difference before and after the replacement, also known as average permutation accuracy difference.

Text analysis refers to the processing of large-scale document sets and the extraction of implicit knowledge from text data sets. Sentiment analysis is a research and application method that uses computational linguistics rules 
to extract emotion words from text. The key areas of text analysis include feature extraction, subject index, clustering and abstract. Text analysis extracts patterns from natural language text and can be defined as the analytical process of extracting information from text according to specific objectives. It combines rules of data mining, information extraction, information retrieval, text classification, probabilistic model, linear geometry, machine learning and computational linguistics to discover the structure, pattern and knowledge of text sets.

Based on computational linguistics and statistical mathematical analysis, text analysis combines machine learning and information retrieval techniques to discover and extract implicit knowledge in document sets independent of users' information needs. It is a process from text information description to selection and extraction mode, and finally form user understandable information knowledge.

\section{Analysis of Residents' Satisfaction with Waste Classification Based on Descriptive Statistics}

We interest from the policies and regulations, management, legislative support and daily waste sorting initiative measures in three aspects: the Beijing residents satisfaction of waste classification, and USES the principle of five-point Likert scale score, among them 1 points represent very disagree, five points represent very identity, the higher the score indicates that residents satisfaction they sort the waste, the better.

According to the results, the overall mean value of satisfaction with waste classification effect was 3.78 , and the variance was 0.978 , indicating that residents' satisfaction with waste classification was relatively high and evenly distributed. In terms of each dimension, the mean value of support for waste classification management legislation is 4.02, which is the highest among the three dimensions, indicating that residents strongly support waste classification legislation. The mean value of attention to policies and regulations was 3.40 , the lowest among the three dimensions, indicating that residents paid general attention to relevant policies and regulations.

Table 3. Waste classification effect satisfaction of all dimensions.

\begin{tabular}{lll}
\hline Category & Average & Variance \\
\hline Attention of Policies and regulations & 3.4 & 1.013 \\
Manage legislative support & 4.02 & 0.949 \\
Daily classification initiative & 3.93 & 0.971 \\
Total & 3.78 & 0.978 \\
\hline
\end{tabular}

After further investigation on the active intention of waste classification in the daily life of Beijing residents, the results are shown in Figure 1 below. $46.98 \%$ and $28.84 \%$ of the investigated Beijing residents are satisfied with and very satisfied with the waste classification effect in their daily life. While the remaining $16.91 \%, 3.98 \%$ and $3.29 \%$ residents held a general attitude towards the satisfaction of waste classification effect in daily life, or even were very dissatisfied or dissatisfied.

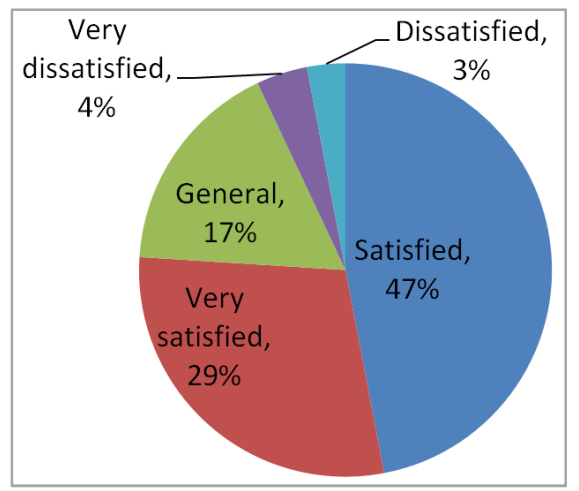

Figure 1. Satisfaction degree of daily household waste classification of Beijing residents.

As can be seen from the above analysis, more than $70 \%$ of residents are satisfied with and very satisfied with the implementation effect of waste classification in their daily life. However, other residents in their daily life are generally or even very dissatisfied with the effect of waste classification. Therefore, in order to further investigate the influencing factors of residents' satisfaction with waste classification, this paper uses random forest method for further analysis.

\section{Analysis of Residents' Satisfaction with Waste Classification Based on Random Forest Model}

The machine learning model can be used to conduct binary classification mining on the samples collected from the waste classification questionnaire to determine whether the current residents of Beijing are satisfied with the implementation of the waste classification policy, and the machine learning model can evaluate the importance of different indicators in the questionnaire to people's satisfaction with the final waste classification. Random forest algorithm can be used for multivariate or binary classification. The random forest algorithm is used to evaluate the importance of indicators affecting the effectiveness of waste classification. Because the random forest algorithm can avoid dimension disaster to a certain extent, its classification performance is better than that of classification regression decision tree CART algorithm alone.

To use machine learning methods to collect samples for binary classification mining waste classification and indicators of questionnaire test policy impact on residents' waste sorting effect satisfaction degree, can effectively use and to extract the characteristics of existing data contains new features, for Beijing to waste classification decision continuously provide valuable advice. The research shows that many traditional machine learning algorithms, such as decision tree, naive Bayes, support vector machine and neural network, can be effectively applied to waste 
classification index verification. Although some features can be used to explain residents' satisfaction with waste classification policies, if all the features are directly used in the training of these traditional classifiers at one time, it is highly likely to lead to low performance and low accuracy of classifiers due to "dimension disaster" and other reasons.

\subsection{Model Selection}

We selected the currently commonly used machine learning classification algorithm to explore whether residents are willing to carry out waste classification. These methods include Logistic regression (LR), support vector machine (SVC), decision tree (DT), random forest (RF) and gradient satisfaction 47\% Very Satisfied 29\% General 17\% Very Dissatisfied 4\% Dissatisfied 3\% improvement (XGBoost).

Standard indexes commonly used in machine learning and information retrieval were used as evaluation indexes in this study, including Accuracy, Precision, Recall, F1 value and AUC score, to measure the classification effects of different machine learning models on the satisfaction of waste classification in Beijing. Accuracy refers to the proportion of all samples with correct prediction (positive or negative) in the total sample.

$$
\text { Accuracy }=\frac{T P+T N}{T P+T N+F P+F N}
$$

Precision refers to the proportion of samples with positive predictions to all samples with positive predictions.

$$
\text { Precision }=\frac{T P}{T P+F P}
$$

Recall refers to the proportion of correctly predicted positive samples in all actual positive samples.

$$
\text { Recall }=\frac{T P}{T P+F N}
$$

The value of F1 is the arithmetic mean of the sample divided by the geometric mean.

$$
F_{1}=\frac{2 P R}{P+R}=\frac{2 T P}{2 T P+F P+F N}
$$

AUC value is the area under the ROC curve, a comprehensive indicator reflecting the continuous variables of sensitivity and specificity. Before training evaluation index, because the goal is to explore the influence factors of Beijing residents waste classification effect of satisfaction, so we will sample based information index of the data set (gender, age, education level, living area and other six indicators) to eliminate, leaving the rest 12 indicators associated with residents satisfaction they sort the waste, concrete are shown in table 4 below:

Table 4. Selected 12 indicators related to residents' satisfaction with the effect of waste classification.

\begin{tabular}{ll}
\hline Indicators & Indicators \\
\hline No classification reason & Publicity and education \\
Propaganda policy & Village view \\
Promote the way & Penalty \\
Classification of cognitive & Classification in the home \\
The implementation of difficult & Propaganda way \\
Group work & Classification evaluation \\
\hline
\end{tabular}

After model evaluation of the five classification algorithms, we obtained the evaluation index results of the five models as shown in the table below:

Table 5. Evaluation index results of five classification models.

\begin{tabular}{lllllll}
\hline & & Accuracy & Precision & Recall & F1-Score & AUC-Score \\
\hline \multirow{2}{*}{ LR } & train & 0.985794 & 0.984081 & 0.999265 & 0.991615 & 0.999348 \\
& test & 0.968345 & 0.962963 & 1.000000 & 0.981132 & 0.993490 \\
SVC & train & 0.990117 & 0.988381 & 1.000000 & 0.994156 & 0.999920 \\
& test & 0.981295 & 0.977778 & 1.000000 & 0.988764 & 0.997299 \\
DT & train & 0.980235 & 0.981173 & 0.995591 & 0.988330 & 0.994642 \\
& test & 0.956835 & 0.962457 & 0.986014 & 0.974093 & 0.938520 \\
RF & train & 1.000000 & 1.000000 & 1.000000 & 1.000000 & 1.000000 \\
& test & 0.955396 & 0.948590 & 1.000000 & 0.973617 & 0.996987 \\
XGB & train & 1.000000 & 1.000000 & 1.000000 & 1.000000 & 1.000000 \\
& test & 0.974101 & 0.975945 & 0.993007 & 0.984402 & 0.997072 \\
\hline
\end{tabular}

According to the evaluation index results in the table, we can clearly see that the five models have shown excellent effects on the classification effect of the satisfaction data set of Beijing residents' waste classification effect. The overall effect of random forest model is the best among the five models. Accuracy, Precision, Recall, F1 and AUC values of its training set are all 1 (the closer the results of the five evaluation indexes are to 1 , the higher the classification Accuracy of the model is). It shows that the prediction result of the model is not different from the real result. Although the evaluation results of the test set are not as good as the XGBoost model, the random forest model has better antioverfitting performance than the gradient promotion model (if all the five evaluation indexes are close to or equal to 1, the model overfitting phenomenon is likely to occur). In addition, the ROC (Receiver operation Characteristic Curve) diagram of random forest can further confirm why we choose random forest model for model prediction and classification, as shown in the figure below:

As can be seen from the figure, the area of ROC curves of both the training set and the test set is close to 1 , which is close to the ideal situation. The true class rate of TPR is close to 1 , and the false positive class rate of FPR is close to 0 . The ROC curve is close to the point $(0,1)$ and deviates from the 45-degree diagonal. Therefore, after the above discussion, we finally selected the random forest model to analyze the 
satisfaction of waste classification effect of Beijing residents.

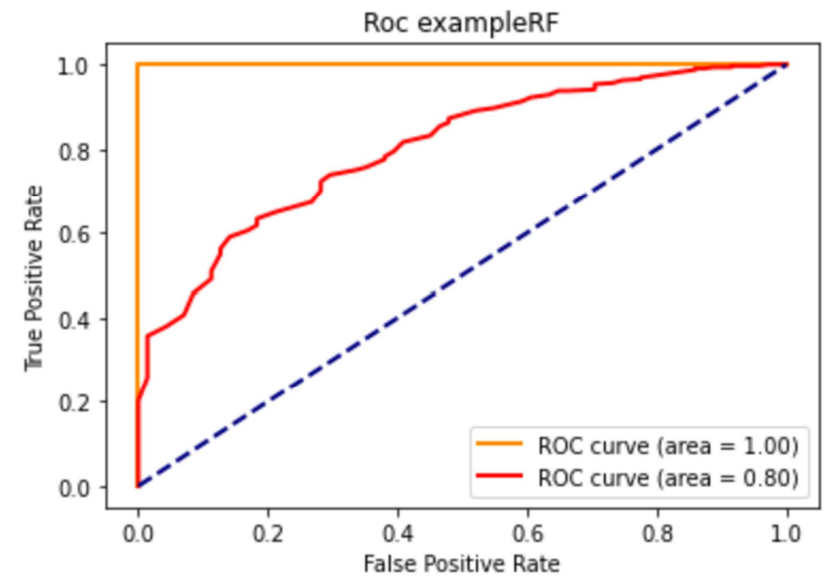

Figure 2. ROC curve of random forest.

\subsection{Analysis of Influencing Factors on Satisfaction of Waste Classification}

Classifying sample data set, we three studies in the sample of Beijing residents waste classification of 7 (you usually pay attention to the Beijing municipal life waste management policies and regulations), 8 (you support Beijing to waste classification management legislation) and 9 (you in daily life, are you willing to take the initiative to classify the waste and) scores averaging, A new field is generated named Category Satisfaction. This field will serve as the y variable in the model, the remaining fields will enter the model as $\mathrm{x}$ variables.

$K$ subsets are extracted from the stress-stress ability training set of college students, and $m$ samples are extracted from the stress ability set $X=\left\{x_{1}, x_{2}, \cdots, x_{n}\right\}$ by using bagging algorithm to construct $K$ subsets of the same size.

(1) Randomly select $F$ features from the original $d$ features, $F=\log _{2} d+1$.

(2) Using formulas, the information gain of $F$ features is calculated respectively, and the feature with the largest information gain is selected as the segmentation attribute of the node.

$$
\begin{gathered}
\Delta_{\text {info }}=\operatorname{Ent}(\text { parent })-\sum_{j=1}^{2} \frac{N(\alpha)}{N} \operatorname{Ent}(\alpha) \\
\operatorname{Ent}(t)=-\sum_{i=1}^{2} p(i \mid t) \log _{2} p(i \mid t)
\end{gathered}
$$

Where $p(i \mid t)$ represents the proportion of node $t$ belonging to category $j, N$ represents the total number of records on the parent node, $N(\alpha)$ Represents a child node $\alpha$ The number of associated records, $j$ indicates the satisfaction category of waste classification, $j=0$ indicates that residents are not satisfied with the waste classification effect, and $j=1$ indicates that residents are satisfied with the waste classification effect.

(3) Repeat the above method to construct each branch of the decision tree.

(4) Repeat (1) - (3) until $K$ decision trees are constructed.

The predictive test data were input into the random forest classifier model, and the satisfaction degree of waste classification was predicted by voting. The final score of the degree of influence on the importance index of residents' waste classification satisfaction is obtained, and the top ten influencing factors are shown as follows:

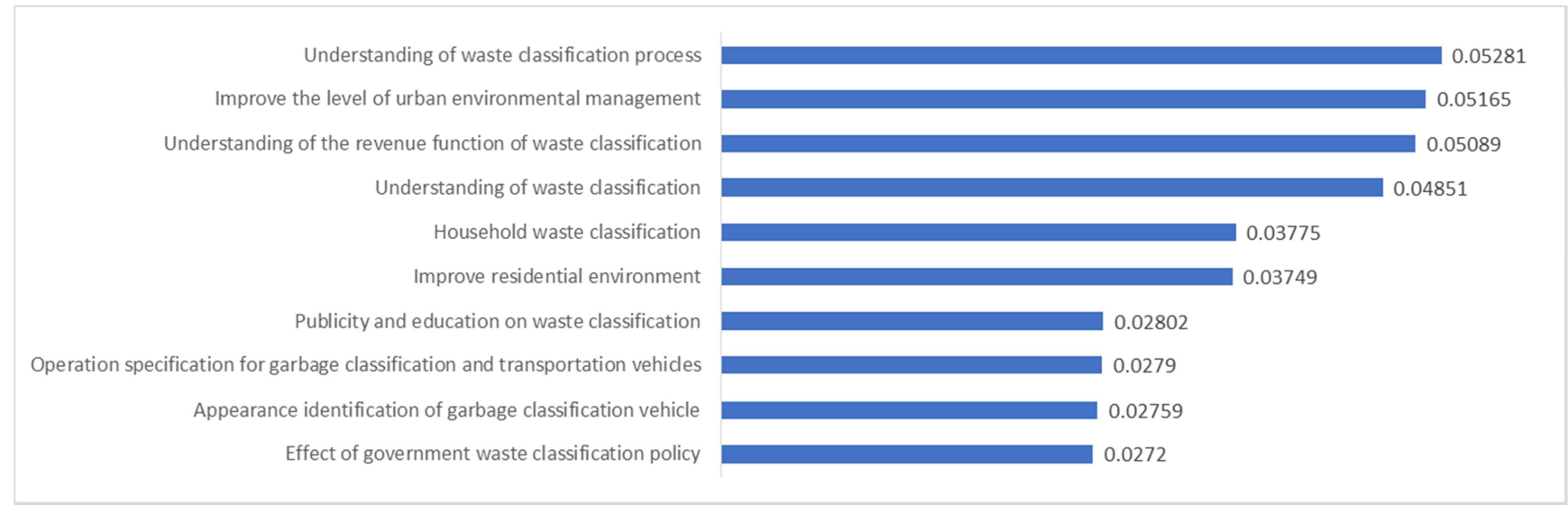

Figure 3. Top 10 factors of residents' satisfaction with waste classification.

As can be seen from the figure, the influencing factors of the top 10 residents' satisfaction with waste classification can be divided into two categories. The first kind can be summarized as Beijing residents' cognitive level of waste classification, residents for waste classification process and benefits, to their active waste classification have a significant impact, in which the government waste classification for residents of publicity and education function also played a promoting residents satisfied with waste sorting effect. In the index importance score obtained by random forest, residents' understanding of waste classification process is 0.052812 , residents' understanding of benefits and effects of waste classification is 0.050889 , residents' understanding of waste classification is 0.048507 , residents' home classification is 0.037745 , and the government's propaganda and education of waste classification is 0.028021 . The total score of these indicators is 0.217974 . Random forest divided the total score 1 into 12 selected indicators, and allocated the score according to the impact of different 
indicators on the satisfaction degree of waste classification of residents set by us. It can be seen that Beijing residents' awareness of waste classification will directly affect residents' satisfaction with waste classification.

The second category can be summarized as residents' evaluation of waste classification policy will also affect residents' satisfaction with waste classification. Waste classification will improve the level of urban environmental management 0.051647 , waste classification will improve the residential environment 0.037487 , waste classification transport vehicle operation specification 0.027904, waste classification vehicle appearance identification 0.027593, government waste classification policy effect 0.027204 . The total score of such indicators is 0.171835 . The effect of waste classification is related to the environment of residents' residential areas. Residents living in a clean environment will promote their satisfaction with waste classification. The total score of the two categories was 0.389809 , accounting for nearly $40 \%$ of the total score of the 12 categories.

It can be seen that residents' knowledge of waste classification and their views on waste classification policies will directly affect residents' satisfaction with waste classification. When the government and communities implement waste classification policies, they should make it easier for residents to understand waste classification knowledge, and popularize waste classification knowledge to residents through relevant community personnel. At the same time, the government should strengthen the supervision on the effectiveness of waste classification facilities and policies, so that residents can truly feel the convenience and benefits of waste classification, and residents will be more satisfied with the effect of waste classification if their evaluation of waste classification is higher.

\section{Review Analysis Based on This Text Mining}

In the process of implementing the waste sorting policy, many Beijing residents have used social media platforms such as Weibo and Tieba to express their feelings. It not only includes residents' evaluation of the implementation of waste classification policy in Beijing, but also their own direct feelings after waste classification experience. By making full use of such information, on the one hand, Beijing residents' satisfaction with waste classification can be found directly, residents' voices can be understood, and future policy plans of the government can be planned. On the other hand, it can also help the government and the community to know how satisfied Beijing residents are with waste classification, laying a good foundation for further policy promotion.

\subsection{The Data Collection}

By comparing various social networking platforms, it is found that more users are concerned about the waste classification policy of Beijing on Weibo than on other platforms, so we choose Weibo for data collection. The basic elements of microblog users' Posting include: user name, number of likes and replies, microblog content and topic name, etc. The data collection work in this paper is completed by Python, and the data collection logic is written and run. Because the program is running need to determine the topic, so we to \# \# Beijing municipal waste classification topic to grab Weibo users sent on waste sorting inscription, in addition, we also to Beijing municipal waste classification for the keyword set hair, time is on May 1, 2020-2021 on March 26, grab the Beijing residents satisfaction for waste classification policy review.

In this paper, 1068 pieces of data were captured successfully by Python. In the process of data capture, unstructured text was transformed into visual structured data and stored in excel form.

\subsection{Data Cleaning and Processing at the Cleaning Place}

In data mining, there are a lot of non-standard and incomplete data in the massive original data obtained through microblog, which will seriously affect the analysis efficiency in the process of subsequent text analysis, and may even lead to the deviation of analysis results. For microblog platforms, there are some phenomena such as users' free speech and punctuation, so it is particularly important to clean and process the original data. The investigation focused on cleaning data that contained letters, numbers, punctuation marks or pictures that were not related to Beijing's waste classification. After data pretreatment, 986 valid data and 82 invalid data were obtained. Further analysis shows that the main reason for the invalid microblog data is that there are a lot of repeated comments in the topic or users' Posting and comments have nothing to do with the waste classification topic of Beijing. We screened out these invalid data.

\subsection{Formation of High Frequency Thesaurus}

After obtaining the data, we use Jieba word segmentation to segment the data corresponding to each field. After processing, we import the word segmentation text into Python to mine effective information and form a highfrequency thesaurus, so that visitors can understand the main idea at a glance. The high-frequency thesaurus is as follows:

Table 6. A list of the top ten high-frequency words for residents' satisfaction with waste classification.

\begin{tabular}{ll}
\hline Words & Word frequency \\
\hline Propaganda & 283 \\
Efforts to & 107 \\
Community & 74 \\
Consciousness & 63 \\
The trash & 62 \\
Management & 54 \\
Facilities & 48 \\
Improve & 47 \\
Reward & 38 \\
Residential & 38 \\
\hline
\end{tabular}

Through further analysis of the data, we can find the implicit relationship between each word of the highfrequency thesaurus and the word cloud: First, the low 
satisfaction degree of waste classification of residents is directly related to the insufficient publicity efforts of waste classification policies of communities; Second, the damage or absence of waste sorting facilities in communities, such as waste cans, is also the reason why residents are not satisfied with the effect of waste sorting. Thirdly, most residents suggest that Beijing residents' waste classification policy should be implemented in combination with incentives, and incentives and support should be provided to residents who conduct waste classification, so that more people can improve their satisfaction in the waste classification process.

According to the above analysis, reasonable incentive policies of rewards and punishments can help improve residents' satisfaction with waste classification. Therefore, we conducted text mining on the subjective suggestions in the questionnaire to obtain residents' suggestions on the implementation of waste classification.

\subsection{Residents' Opinions on the Reward and Punishment Measures of Waste Classification Policy}

After preliminary observation of residents' comments on the policy of rewarding and punishing waste classification, we added the word 'Doukeyi' to the list of stop words. At the same time, in order to avoid the appearance of reward and punishment, which would lead to the loss of analytical significance and value of the final analysis results, we also added these two words into the stop words database. The stop words selected during the analysis of residents' waste classification behavior should be reserved. After word frequency analysis of residents' comments on waste classification policies, we obtained the top five words that appeared frequently in residents' attitudes towards waste classification policies, as shown in the figure below:

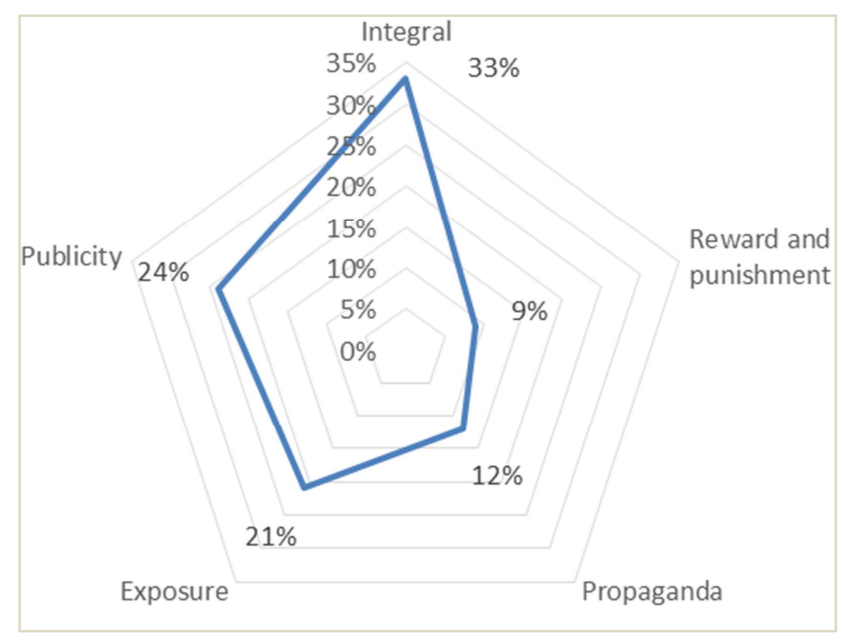

Figure 4. The top five words of attitude of residents' waste sorting policy, reward and punishment measures appeared frequently.

It can be seen from the results in the figure that the incentive method for waste classification advocated by residents is to collect individual points after waste classification and exchange them for rewards through points. The frequency of the word "integral" was 33\%, which was the word with the highest frequency among all the residents' attitudes towards the reward and punishment methods of waste classification. "Exposure" was used in $21 \%$ of residents' attitudes towards punishing those who failed to separate waste. The remaining residents gave suggestions on how to implement the waste sorting policy in terms of rewards and punishments, with the frequency of publicity being $24 \%$, publicity being $12 \%$, and both rewards and punishments being $9 \%$. From the residents' point of view, as long as the community can publicize the waste classification policy in a timely manner and reward and punishment are clear, residents' satisfaction with waste classification can be promoted.

\subsection{Residents' Opinions on the Difficulties in Implementing the Waste Sorting Policy}

In the implementation of waste sorting policies, various difficulties will be encountered, but these difficulties are difficult to be perceived by the policy implementers. Only through the reflection attitude of each resident who personally participated in waste classification can we find out what kind of difficulties encountered in the real implementation of waste classification. The use of waste classification behavior performance and residents for waste classification policy reward and punishment methods have been set to stop the word table. In order to avoid the phenomenon of meaningless analysis after the final word segmentation, the word difficult is added to the stop word list. After word frequency analysis of residents' comments on the difficulties in implementing waste classification policy, we obtained the top five words that appeared frequently in residents' comments on the difficulties in implementing waste classification policy, as shown in Figure 5.

From the top five words chart of residents' attitudes towards difficulties in implementing waste sorting policies, we can see that the lack of policy implementation is the primary problem causing difficulties in implementing waste sorting policies, with the frequency of "lack" being $24 \%$. The lack of rewards and punishments for residents' waste classification behavior is a secondary problem that causes difficulties in implementing waste classification policies. The frequency of rewards and punishments is $22 \%$, and the frequency of punishment is $19 \%$. In addition, the nonstandard implementation of waste classification policy and the adverse supervision of community managers and government on residents' waste classification are also the key to the difficult implementation of waste classification policy. The word "regulation" was used $19 \%$ of the time and "supervision" was used $17 \%$ of the time. The government and communities should focus on solving the problems of weak publicity and lax supervision when carrying out waste sorting policies, and at the same time introduce reward and punishment policies timely to make waste sorting residents more satisfied with the classification effect. 


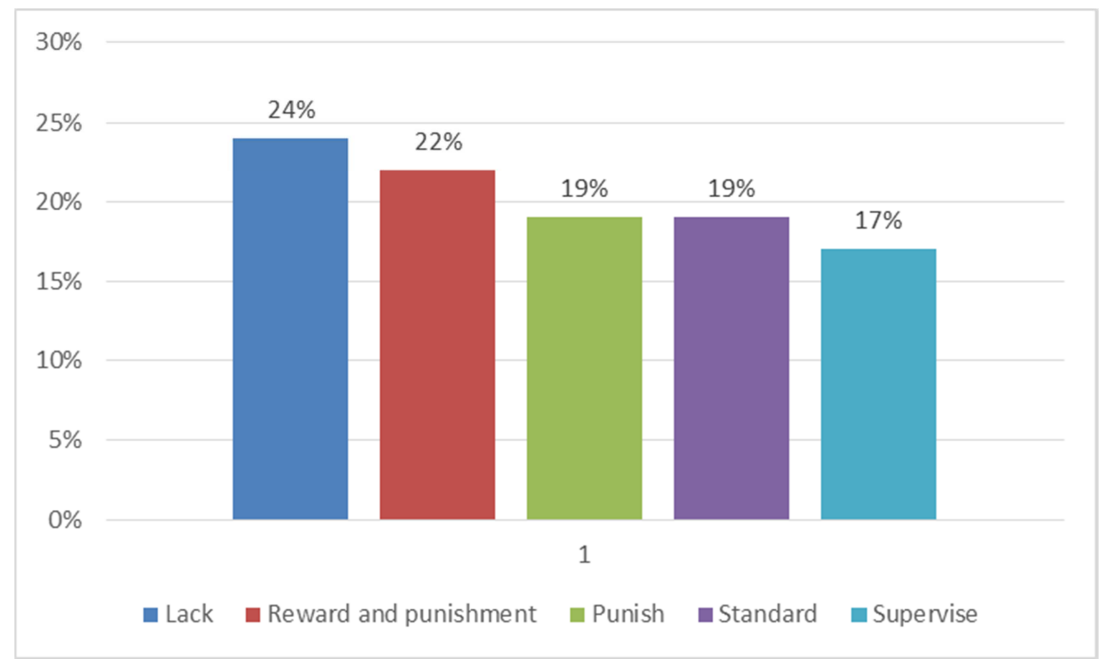

Figure 5. The top five words of residents' attitude about difficulties in implementing waste sorting policies appeared frequently.

\section{Conclusions and Recommendations}

\subsection{Conclusions}

Based on the above analysis, we know that residents' satisfaction with waste classification is generally at a medium level, with the highest satisfaction with the government's environmental management level and the worst satisfaction with the effect of policy implementation. In addition, the satisfaction degree of different characteristic groups is also different, and the willingness of waste classification, the degree of process standardization and the degree of classification cognition complement each other to affect residents' satisfaction. The more irregular the waste classification process is in the residential area, the less profound the recognition of waste classification policy will affect the residents' willingness to carry out waste classification, and ultimately affect the satisfaction of waste classification. It is gratifying that Beijing residents hold a positive attitude towards the implementation of waste classification policy, and most of them believe that the implementation of reward and punishment policies can improve residents' satisfaction with the implementation of waste classification policy.

In conclusion, we find that the implementation of reward and punishment laws in the process of waste classification policy not only affects the implementation effect of waste classification policy, but also affects residents' satisfaction with the implementation of waste classification policy. $24 \%$, $22 \%, 19 \%, 19 \%, 17 \%, 0 \%, 10 \%, 15 \%, 20 \%, 25 \%, 30 \%$ lack the supervision of reward and punishment standards and have a positive impact on the satisfaction of waste classification policy implementation. At the same time, the standardization degree and cognition degree of waste classification process in residential areas have a profound impact on residents' intention of waste classification, and have a direct impact on the implementation effect of waste classification policy and residents' satisfaction.

\subsection{Recommendations}

\subsubsection{Establish and Improve the Long-term Publicity and Guidance Mechanism}

Carry out various forms of waste classification publicity activities, carry out extensive propaganda and education on why waste should be classified and how to classify it. At the same time, create a strong public opinion atmosphere, broaden the medium of category classification policies and procedures, through the living waste classification knowledge propaganda film making, public service ads, instruction manual, etc., to employ environmental image ambassador, through newspapers, radio, television, network, a variety of forms such as bus mobile media to carry out publicity and education, improve the ideological understanding of the masses.

\subsubsection{Establish Scientific Operation Mechanism}

Perfect foundation facilities construction waste classification, which requires the local policy makers with community manager coordinate with each other, the government is responsible for purchasing enough quantity carries different classified waste facilities, community can choose the most convenient to residents of waste on the site, let people no longer because the lack of facilities complete or not, give up the existing waste sorting into consciousness, Appear to have sorted waste mixed into the waste can resulting in inefficient classification results.

\subsubsection{We Will Establish a Multi-form Interest-oriented Mechanism}

To take a certain incentive and punishment mechanism for residents, according to whether individuals participate in waste classification, the frequency of waste delivery to establish different charging standards, with economic leverage to promote source reduction and waste classification work. Promote the construction of waste classification points exchange system, establish a red and black list based on commendation and reward for families and individuals who 
participate in waste classification activities with outstanding performance, and reasonable punishment for groups that do not carry out waste classification.

\subsubsection{Establish an Effective Guarantee Mechanism}

We will establish and improve waste classification laws, regulations and management agencies, and improve the comprehensive departmental coordination mechanism. Waste classification should be mandatory by laws and regulations to ensure the long-term effectiveness of waste classification work, so that waste classification placement, collection, transportation, resource utilization, harmless disposal and other links into the track of legal management, establish hard standards, adhere to the gradual progress. At the same time, it is necessary for all departments to cooperate and promote the establishment of long-term mechanism of waste classification under multi-pronged efforts, so as to achieve the pattern of "co-construction, co-governance and sharing".

\section{Funding}

The research is supported by Analysis of Course Examination Results and Comprehensive Evaluation of Teaching Effect.

\section{References}

[1] Li Wei, Wang Zhihao, Liu Xiaoguang. Influence mechanism of publicity and education on urban residents' intention of waste classification - The mediating role of environmental emotion and the moderating role of moral identity $[\mathrm{J}]$. Arid land resources and environment, 2021, 35 (03): 21-28.

[2] Hu Changchun, Ning Changhui. For "I" or "we"? - Research on the influence of different types of waste classification propaganda on waste classification behavior intention [J]. Prediction, 2021, 40 (02): 9-16.

[3] Chen Jian, Lin Weibin, Li Yujun. An empirical study on the willingness and behavior of waste classification - Take Guangzhou as an example [J]. City watch, 2020 (01): 133-143.
[4] Xu Qianqian, Wang Chengjun, Zhang Shuhe. Influence of household participation on the effect of rural household waste classification and treatment $[\mathrm{J}]$. Journal of Agricultural Resources and Environment, 2021, 38 (02): 223-231.

[5] Wang Qiqi. Effect evaluation and development strategy of Waste classification in China - Take Shanghai universities as an example [J]. Market weekly, 2020, 33 (10): 127-130.

[6] Zhao Junqing. Exploration of application of standard curve method to effect evaluation of household waste classification [J]. Comprehensive utilization of resources in China, 2021, 39 (01): 51-55.

[7] Musee, L. Lorenzen, C. Aldrich. New methodology for hazardous waste classification using fuzzy set theory Part I. Knowledge acquisition [J]. N. Musee et al. / Journal of Hazardous Materials 154, 2008, 1040-1051.

[8] Seong-Rin Lim, Daniel Kang, Oladele A. Ogunseitan, Julie M. Schoenung. Potential Environmental Impacts of LightEmitting Diodes (LEDs): Metallic Resources, Toxicity, and Hazardous Waste Classification [J]. Environ. Sci. Technol, 2011, 45, 320-327.

[9] R. Weltens, K. Deprez, L. Michiels. Validation of Microtox as a first screening tool for waste classification [J]. Waste Management, 2014.

[10] Qu Xiaoyan, Zhang Linnan, Yang Lei, Li Zhenshan. Beijing municipal Solid waste classification and treatment system Problem diagnosis [J]. Environmental pollution and prevention, 2009, (05).

[11] Deng Jun, Xu Wanying, Zhou Chuanbin. Research on the effective investigation and long-term management mechanism of sorted collection of Municipal solid waste in Beijing [J]. Environmental science, 2013, (01).

[12] Wang Chaohua. The problems of waste classification in Beijing area are analyzed and the countermeasures are suggested [J]. Economic, 2018, (02).

[13] Yu Dian, Wang Chong, Shi Ruyue, Yang Junxue. Study on the present situation and countermeasures of rural MSW treatment in Beijing $[\mathrm{J}]$. Modern rural science and technology, 2020, (08). 